Article

\title{
Current Evidence on the Antimicrobial Treatment and Chemoprophylaxis of Human Leptospirosis: A Meta-Analysis
}

\author{
Marta Guzmán Pérez ${ }^{1}$, José Javier Blanch Sancho ${ }^{2}$, Juan Carlos Segura Luque ${ }^{2,3}$, Fernando Mateos Rodriguez ${ }^{2}$, \\ Elisa Martínez Alfaro ${ }^{2}$ and Julián Solís García del Pozo 2,3,*iD \\ 1 Department of Internal Medicine, Albacete University Hospital, 02006 Albacete, Spain; \\ martaguzpe661@gmail.com \\ 2 Infectious Diseases Unit, Department of Internal Medicine, Albacete University Hospital, \\ 02006 Albacete, Spain; jjblanchs@sescam.jccm.es (J.J.B.S.); jcsegural@sescam.jccm.es (J.C.S.L.); \\ fmateosrodriguez@gmail.com (F.M.R.); elisamartinezalfaro@gmail.com (E.M.A.) \\ 3 Department of Medical Sciences, Albacete School of Medicine, University of Castilla La Mancha, \\ 02008 Albacete, Spain \\ * Correspondence: julianeloysolis@gmail.com
}

Citation: Guzmán Pérez, M.; Blanch Sancho, J.J.; Segura Luque, J.C.; Mateos Rodriguez, F.; Martínez Alfaro, E.; Solís García del Pozo, J. Current Evidence on the Antimicrobial Treatment and Chemoprophylaxis of Human Leptospirosis: A Meta-Analysis. Pathogens 2021, 10, 1125. https:// doi.org/10.3390/pathogens10091125

Academic Editors: Michael J. Maze and Jackie Benschop

Received: 10 August 2021

Accepted: 31 August 2021

Published: 2 September 2021

Publisher's Note: MDPI stays neutral with regard to jurisdictional claims in published maps and institutional affiliations.

Copyright: (c) 2021 by the authors. Licensee MDPI, Basel, Switzerland This article is an open access article distributed under the terms and conditions of the Creative Commons Attribution (CC BY) license (https:// creativecommons.org/licenses/by/ $4.0 /)$.

\begin{abstract}
Background: Leptospirosis is a worldwide zoonotic infection, and its management needs to be refined. This study aims to discern which antibiotic would be the best option to treat leptospirosis disease and analyze the efficacy of chemoprophylaxis regimens to prevent this illness. Methods: systematic review and meta-analysis on the efficacy of antibiotic treatment and chemoprophylaxis of leptospirosis in humans. Results: Ten clinical trials compared an antibiotic treatment with placebo or other antibiotic treatments in leptospirosis (the most recent one was published in 2007). The meta-analysis shows no effect of penicillin treatment on mortality compared to placebo (OR 1.65; 95\% CI 0.76-3.57; $p=0.21$ ). There are no differences between penicillin and cephalosporins or doxycycline. Penicillin does not reduce the time of defervescence (MD-0.16; 95\% CI (-1.4) -1.08; $p=0.80)$ nor hospital stay (MD 0.15; 95\% CI $(-0.75)-1.06 ; p=0.74)$. Besides, the data did not demonstrate any effectiveness of the use of penicillin in terms of the incidence of oliguria/anuria, the need for dialysis treatment, time to creatinine normalization, incidence of jaundice, or the liver function normalization time. Eight trials have assessed prophylactic treatment against leptospirosis with different strategies. A weekly dose of $200 \mathrm{mg}$ of doxycycline does not show benefit versus placebo regarding the number of new cases of symptomatic leptospirosis (OR 0.20; 95\% CI 0.02-1.87; $p=0.16$ ). A single dose of doxycycline at exposure to flood water could have a beneficial effect (OR $0.23 ; 95 \%$ CI $0.07-0.77 ; p=0.02$ ). None of the other chemoprophylaxis regimens tested have shown a statistically significant effect on the number of new symptomatic cases. Conclusion: There is no evidence that antibiotics are a better treatment than placebo regarding mortality, shortening of fever, liver and kidney function, or reduction in the hospital stay. On the other hand, neither doxycycline nor penicillin, nor azithromycin have shown statistically significant differences in preventing symptomatic infection. Well-designed clinical trials, including other antibiotics such as quinolones or aminoglycosides, are urgently needed to improve our understanding of the treatment for this infection, which continues to be a neglected disease.
\end{abstract}

Keywords: Leptospirosis; treatment; antibiotics; chemoprophylaxis; systematic review

\section{Introduction}

Leptospirosis is a worldwide zoonotic infection, although its incidence is greater in tropical regions. Leptospira infects more than a million people annually, with approximately 60,000 deaths, and the number of fatal cases is comparable to some other important neglected tropical diseases such as severe dengue or visceral leishmaniasis [1]. In addition, the epidemiology of leptospirosis has been modified by changes in animal husbandry, 
climate, and human behavior [2]. Individuals living in urban slum environments characterized by inadequate sanitation and poor housing are at high risk of exposure. The global burden of leptospirosis is expected to rise with the increment in urban poor in tropical regions [3].

Most cases have a biphasic clinical presentation: a septicemic phase followed by immune manifestations [4]. In the initial phase, the clinical symptoms are not specific and can mimic a flu-like syndrome, making diagnosis often difficult. Nevertheless, some clinical (muscle pain, cough, conjunctival involvement, and jaundice) and biological features (thrombocytopenia, cholestasis, rhabdomyolysis, and a rise in serum $\mathrm{C}$ reactive protein) can help to diagnose leptospirosis and lead to a quick antibiotic therapy before the progression to a severe icterohemorrhagic (Weil's disease) or respiratory form associated with higher mortality [5].

Management of leptospirosis needs to be refined [6,7]. Delays in diagnosis due to the lack of adequate clinical suspicion, its non-specific symptoms, limited availability of rapid point-of-care diagnostic tests are some of the reasons why its mortality remains significant [7]. There are several diagnostic methods, including direct and serological tests. Serological tests are based on the detection of antibodies against leptospiral antigens. A microscopic agglutination test (MAT) would not be useful in the first stages of the disease when antibodies are not present [8]. Direct diagnostic methods include phase contrast or dark field microscopy, histochemical staining and immunostaining, culture methods, and polymerase chain reaction (PCR) [8].

In regions where leptospirosis is endemic, outbreaks might happen following heavy rainfall (e.g., after cyclones) and floods. The distribution of leptospirosis cases is known to be uneven, and regions of highest incidence have been identified in several contexts [9].

Preventive measures should limit the mammal reservoir and human exposure. Moreover, there is a need to improve the underlying environmental conditions and infrastructure deficiencies in urban slum communities to fight against this neglected disease [10].

Chemoprophylaxis has been considered as a way to protect humans from leptospirosis [9]. Penicillin G sodium (penicillin G) is generally recommended for severe leptospirosis [11]. Nevertheless, other antibiotics such as Doxycycline or Ceftriaxone have been proposed as alternatives to penicillin G [11]. This study aims to discern which antibiotic would be the best option to treat leptospirosis disease and analyze the efficacy of chemoprophylaxis regimens to prevent this illness. We also try to identify existing knowledge gaps on this topic. For this purpose, a systematic review of the treatment and chemoprophylaxis of human leptospirosis has been carried out.

\section{Material and Methods}

\subsection{Search Strategy}

A search of all clinical trials comparing any antibiotic for treatment or chemoprophylaxis against human leptospirosis with another antibiotic or placebo until 26 July 2021 was performed. A search was also carried out for trials that looked for the use of antimicrobials for the prophylaxis of Leptospira spp. infection in humans. Studies were identified by searching in MEDLINE, Scopus, and Web of Science. The MEDLINE search used the terms "leptospirosis," "human," "treatment," "therapy," "chemoprophylaxis," "clinical trial [MesH Terms]," "clinical trial as a topic [MeSH Terms]," "clinical trial," "trial." Scopus and Web of Science search used the same terms. MEDLINE search yielded 67 articles. Search in Scopus and Web of Science yielded 99 and 123 articles, respectively. The selected reports' bibliographic references were also examined in search of other possible publications not found in the databases mentioned above. When duplicates were removed, 207 articles were screened. Figure 1 shows the search results and the study flow diagram. 


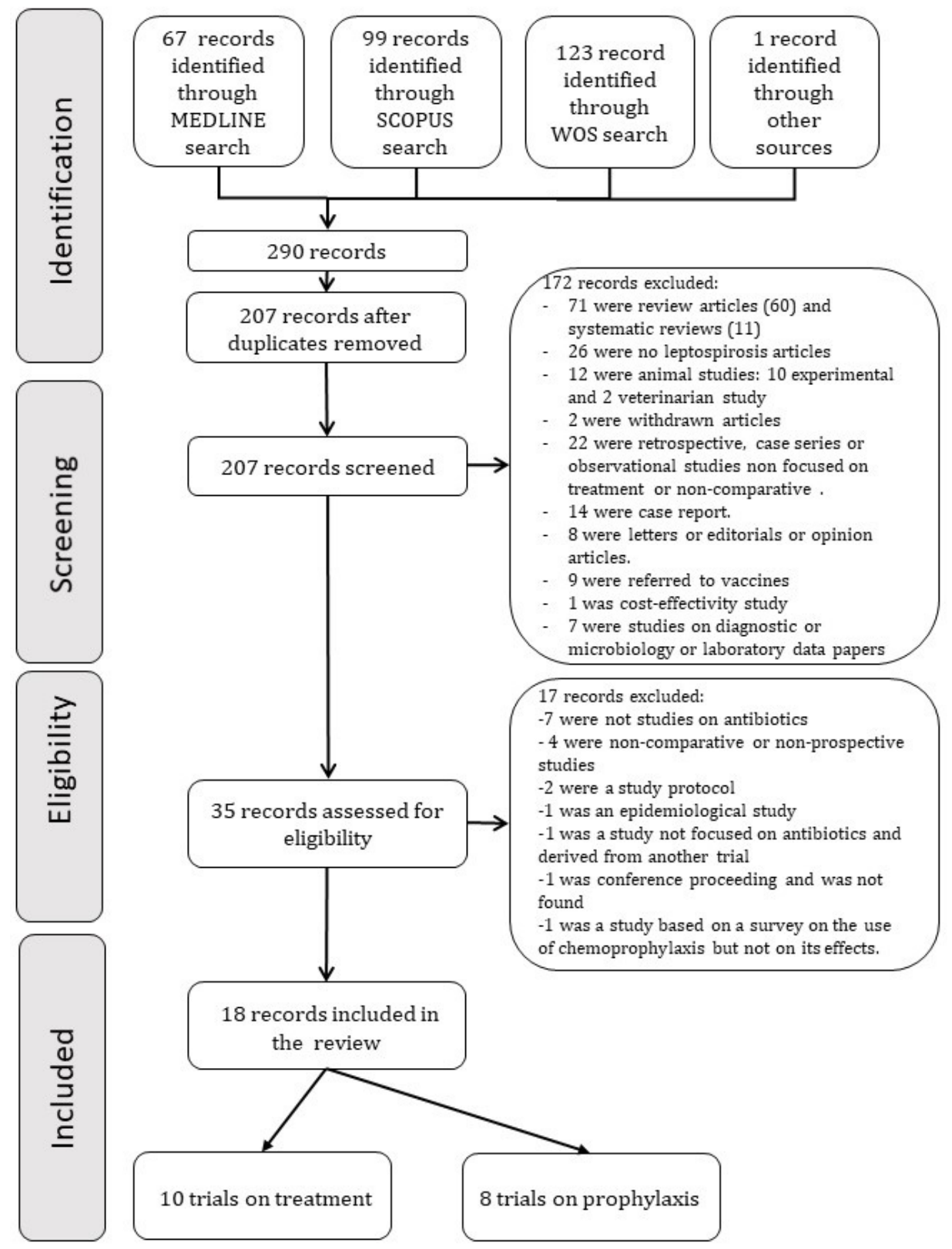

Figure 1. Flow chart of study selection protocol.

\subsection{Selection Criteria}

Clinical trials comparing any antibiotic treatment against human leptospirosis with another antibiotic or placebo or trials looking for the use of any antimicrobial for the prophylaxis of Leptospira spp. infection in humans were analyzed. The studies on treatment should meet the following inclusion criteria: (i) well-characterized patients in terms of diagnosis of leptospirosis, (ii) studies should provide data on the treatment administered, duration, and the dose of antimicrobials used and, (iii) well-defined patient outcome. Studies on the prophylaxis of human leptospirosis should meet the following inclusion criteria: (i) the drug administered should be clearly stated as well as the dose and time of administration, (ii) follow-up time should be specified, (iii) it should include the diagnostic criteria for new cases of leptospirosis. Search and selection of studies were performed independently by two investigators (MGP and JSGP).

Those studies without clear diagnostic criteria and methods were excluded. Other exclusion criteria were: studies that did not show in sufficient detail the drug used, the dose, 
or the duration of treatment. Case reports, case series, non-prospective or non-comparative studies were excluded too, and articles written in languages other than English or Spanish.

\subsection{Outcome Measures}

The parameters considered in the evaluation of the different therapeutic regimens were:

- Duration of hospitalization, defervescence time, kidney failure, liver failure, recovery time from kidney or liver failure, and death;

- For prophylaxis trials, the rate of new cases of leptospirosis in each group was collected.

\subsection{Data Extraction}

Data from studies included in the review were independently extracted by two investigators (MGP and JSGP). Discrepancies were discussed and resolved by consensus. The following variables were collected for each study:

- Author and year of publication;

- Type of study;

- Number of patients;

- Diagnostic criteria;

- Treatment regimens with antibiotics, with its duration, route of administration, and dose;

- If the antibiotic treatment was started as prophylaxis or treatment for the infection;

- Duration of hospitalization, time of defervescence, the incidence of renal or liver failure, recovery time from renal or liver failure, and mortality.

\subsection{Quality Assessment}

Randomized clinical trials were assessed by using the Cochrane Risk of Bias Tool for Randomized Controlled Trials [12] independently by two investigators (MGP and JSGP) (Figure 2). Using this method, the risk of different possible biases has been assessed for each included study, and it has been classified as low risk, high risk, or unclear risk of bias [12].

\subsection{Data Synthesis}

The analysis compared the various therapeutic regimens in terms of hospitalization length of stay, time to defervescence, the incidence of renal or liver failure, recovery time from renal or liver failure, and mortality. For prophylaxis trials, the rate of new symptomatic infections was compared among both comparison groups. The differences between the two regimens compared in each case are expressed as an odds ratio with the confidence interval (CI95\%) and were contrasted using the Mantel-Haenszel test, using a random effect model which assumes that the effects in the different studies are not identical, treating the differences as random. For continuous variables, the mean difference was used as a measure of effect. Cochran's Q statistic and the I2 inconsistency statistic were used to measure heterogeneity regarding study results. In all statistical tests, the level of statistical significance used was $p<0.05$. Analyses were performed using RevMan version 5.4 (The Cochrane Collaboration). 

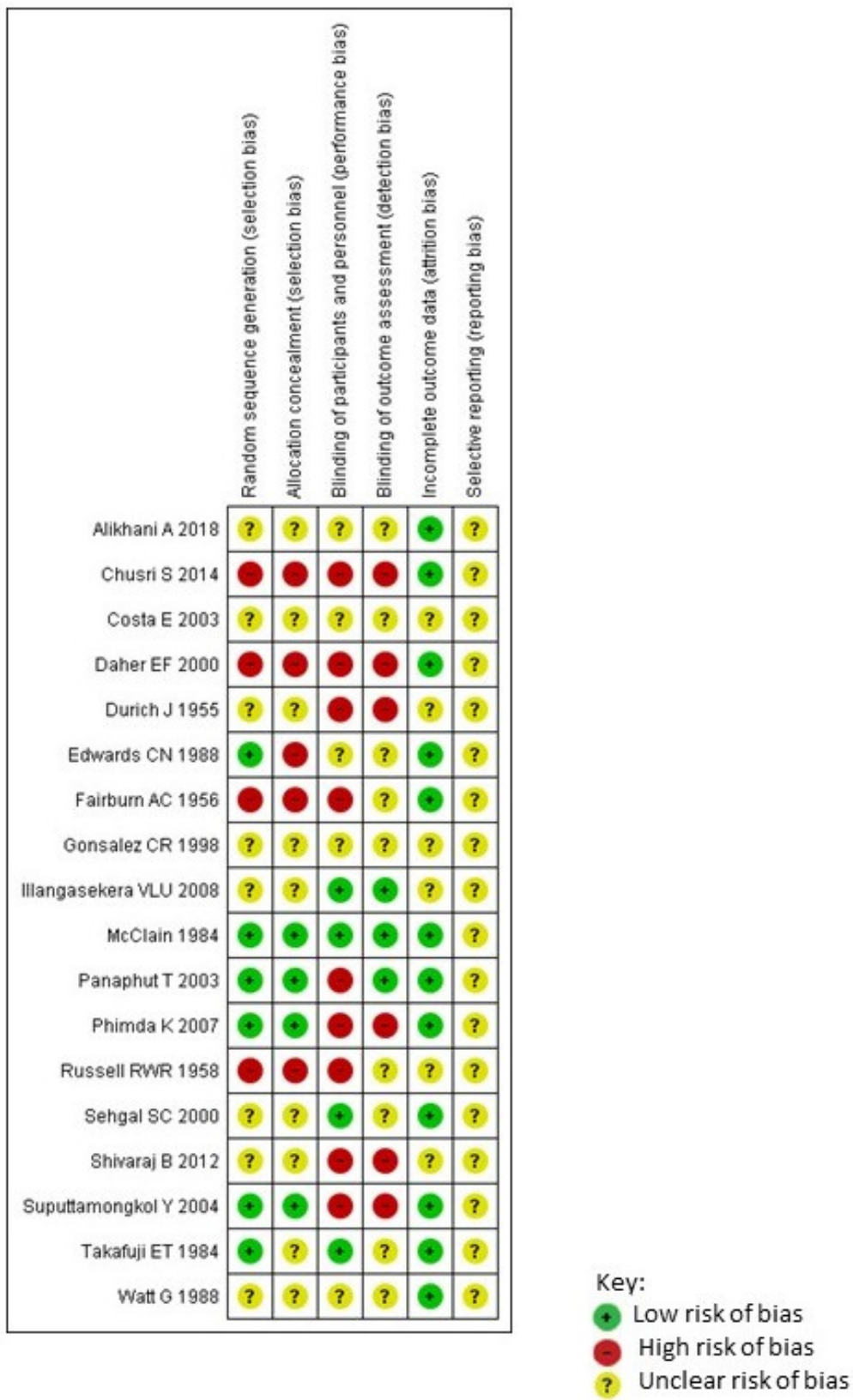

Figure 2. Risk of bias summary: review authors' judgments about each risk of bias item for each included study.

\section{Results}

Ten clinical trials compared an antibiotic treatment with placebo or other antibiotic treatments in leptospirosis [13-22]. Five of them compared penicillin versus placebo or no antibiotic treatment [13,16-19]. Other trials compared penicillin with ceftriaxone [20], doxycycline with placebo [15], oxytetracycline with placebo [14], penicillin with cefotaxime, and doxycycline [21], and doxycycline with azithromycin [22]. The most recent trial on treatment was published in 2007 [23]. Among all these trials, they comprised 1071 patients, of whom 396 received penicillin, 168 received a third-generation cephalosporin, 136 received doxycycline, 35 received azithromycin, 31 chloramphenicol, 27 oxytetracycline, and 278 received no antibiotics (178) or received a placebo (100) (Table 1). 
Table 1. Studies included in the present review.

\begin{tabular}{|c|c|c|c|c|c|c|}
\hline Author (Year) and Reference & Treatment/Prophylaxis & Type of Study & Patients & Treatment Groups & $n$ & Outcomes \\
\hline Fairburn AC (1956) [13] & Treatment & Controlled trial & $\begin{array}{l}\text { Soldiers admitted to one of two } \\
\text { military hospitals in Malaya with } \\
\text { agglutination test positive. }\end{array}$ & $\begin{array}{l}\text { Penicillin } 600,000 \mathrm{UI} / 6 \mathrm{~h} \\
\text { versus chloramphenicol } 0.5 \\
\mathrm{~g} / 6 \mathrm{~h} \text { versus no antibiotics } \\
\text { (for at least five days) }\end{array}$ & 21 vs. 31 vs. 31 & $\begin{array}{c}\text { Duration of fever } \\
\text { Duration of symptoms } \\
\text { "After-peaks" of pyrexia } \\
\text { Complications: Jaundice, } \\
\text { oliguria, anuria, and uremia }\end{array}$ \\
\hline Ross Russell RW (1958) [14] & Treatment & Placebo-controlled trial & $\begin{array}{l}52 \text { consecutive patients admitted } \\
\text { to the military hospital with } \\
\text { leptospirosis confirmed by } \\
\text { blood-culture or serological tests. }\end{array}$ & $\begin{array}{c}\text { Oxytetracycline } 1.5 \mathrm{~g} \text { followed } \\
\text { by } 0.5 \mathrm{~g} / 6 \mathrm{~h} \text { for at least } 5 \text { days } \\
\text { versus placebo }\end{array}$ & 27 vs. 25 & $\begin{array}{c}\text { Duration of pyrexia; } \\
\text { Duration of symptoms; } \\
\text { Incidence of jaundice; } \\
\text { Urea levels. }\end{array}$ \\
\hline McClain (1984) [15] & Treatment & $\begin{array}{c}\text { Randomized, } \\
\text { double-blind controlled } \\
\text { trial }\end{array}$ & $\begin{array}{l}\text { Any febrile patient returning from } \\
\text { jungle training school without } \\
\text { another cause of fever identified } \\
\text { and isolation of leptospira in } \\
\text { blood or urine, or a fourfold rise } \\
\text { in serum antibody titers. }\end{array}$ & $\begin{array}{l}\text { Doxycycline } 100 \mathrm{mg} / 12 \mathrm{~h} \text { for } \\
7 \text { days versus placebo }\end{array}$ & 14 vs. 15 & $\begin{array}{c}\text { Duration of fever; } \\
\text { Duration of signs and } \\
\text { symptoms; } \\
\text { Negativization of } \\
\text { Leptospira in cultures and } \\
\text { MAT. } \\
\end{array}$ \\
\hline Watt G (1988) [16] & Treatment & $\begin{array}{c}\text { Double-blind } \\
\text { placebo-controlled trial }\end{array}$ & $\begin{array}{l}\text { Patients ( }>16 \text { years) with a high } \\
\text { likelihood of leptospirosis and } \\
\text { also have leptospirosis confirmed } \\
\text { with a fourfold or greater rise in } \\
\text { antibody titer by } \\
\text { microagglutination test or } \\
\text { isolation in blood or urine. }\end{array}$ & $\begin{array}{l}\text { Penicillin G } 1.5 \mathrm{MU} / 6 \mathrm{~h} \text { for } 7 \\
\text { days versus placebo }\end{array}$ & 23 vs. 19 & $\begin{array}{l}\text { Duration of fever; } \\
\text { Duration of symptoms. } \\
\text { Duration of hospital stay; } \\
\text { Number of patients from } \\
\text { whom leptospires were } \\
\text { isolated after treatment; } \\
\text { Raised serum creatinine } \\
\text { levels, hepatic tenderness, } \\
\text { and hematological and } \\
\text { biochemical variables. }\end{array}$ \\
\hline Edwards CN (1988) [17] & Treatment & $\begin{array}{l}\text { Randomized controlled } \\
\text { trial }\end{array}$ & $\begin{array}{l}\text { Patients with a history and } \\
\text { physical findings compatible with } \\
\text { symptoms of leptospirosis and } \\
\text { have a fourfold rise in titer or an } \\
\text { initial titer } \geq 600 \text { in the MAT; a } \\
\text { titer } 1: 80 \text { IgM on an ELISA; a } 4+ \\
\text { Patoc I antigen titer; positive } \\
\text { leptospira cultures. }\end{array}$ & $\begin{array}{l}\text { Penicillin } 2 \mathrm{MU} / 6 \mathrm{~h} \text { iv } 5 \text { days } \\
\text { vs. placebo }\end{array}$ & 38 vs. 41 & $\begin{array}{c}\text { Time to defervescence; } \\
\text { Time to return of } \\
\text { biochemical normality; } \\
\text { Absence or positive urine } \\
\text { culture; } \\
\text { Complications; } \\
\text { Mortality; } \\
\text { Iritis. }\end{array}$ \\
\hline
\end{tabular}


Table 1. Cont.

\begin{tabular}{|c|c|c|c|c|c|c|}
\hline $\begin{array}{c}\text { Author (Year) and } \\
\text { Reference }\end{array}$ & Treatment/Prophylaxis & Type of Study & Patients & Treatment Groups & $n$ & Outcomes \\
\hline Daher EF (2000) [18] & Treatment & Probably non randomized ${ }^{\dagger}$ & $\begin{array}{l}\text { Patients with leptospirosis } \\
\text { and acute renal failure } \\
\text { (plasma creatinine }>1.5 \\
\mathrm{mg} / \mathrm{dL} \text { ) and jaundice on } \\
\text { admission. Diagnosis of } \\
\text { leptospirosis clinical ald } \\
\text { IgM } \geq 1: 400 \text { on the } 15 \text { th day } \\
\text { after the onset of symptoms. }\end{array}$ & $\begin{array}{c}\text { Penicillin } 6 \mathrm{MU} / \text { day iv for } 8 \\
\text { days vs. no antibiotics }\end{array}$ & 16 vs. 19 & $\begin{array}{l}\text { Days of hospitalization; } \\
\text { Duration of fever } \\
\text { Time to normalization of } \\
\text { creatinine, bilirubin (or } \\
\text { one-third of maximum } \\
\text { value), and platelet count; } \\
\text { Dialytic treatment; } \\
\text { Values of serum and urine } \\
\text { biochemical parameters; } \\
\text { Hospital mortality; } \\
\text { Presence of oliguria; } \\
\text { Positive fluid balance (\%); } \\
\text { Episodes of bleeding; } \\
\text { X-ray evidence of } \\
\text { pneumonitis. }\end{array}$ \\
\hline Costa E (2003) [19] & Treatment & Randomized controlled trial & $\begin{array}{l}\text { Patients with }>4 \text { days with } \\
\text { symptoms; } \geq 26 \text { points in } \\
\text { the WHO probability score } \\
\text { for leptospirosis; } \\
\text { Macroscopic slide test, } \\
\text { microagglutination test, and } \\
\text { blood culture for } \\
\text { microbiologic confirmation } \\
\text { in Salvador. }\end{array}$ & $\begin{array}{l}\text { Penicillin } 6 \mathrm{mU} / \text { day }(1 \\
\mathrm{mU} / 4 \mathrm{~h} \text { ) for seven days } \\
\text { versus control (no } \\
\text { antibiotic) }\end{array}$ & 125 vs. 128 & $\begin{array}{l}\text { In-Hospital death; } \\
\text { Length of hospital stay. }\end{array}$ \\
\hline Panaphut T (2003) [20] & Treatment & $\begin{array}{l}\text { Randomized trial. } \\
\text { Open-label }\end{array}$ & $\begin{array}{l}\text { Patients (>16 years) with } \\
\text { severe leptospirosis, defined } \\
\text { by the presence of jaundice, } \\
\text { a serum creatine level of } \\
>180 \mu \mathrm{mol} / \mathrm{L} \text {, or a mean } \\
\text { arterial pressure }<70 \mathrm{mmHg} \text {. } \\
\text { Serology diagnostic. WHO } \\
\text { criteria. }\end{array}$ & $\begin{array}{l}\text { Ceftriaxone } 1 \mathrm{~g} / \text { day for } 7 \\
\text { days versus penicillin } \mathrm{G} 1.5 \\
\text { MU } / 6 \mathrm{~h} \text { for } 7 \text { days *. }\end{array}$ & 87 vs. 86 & $\begin{array}{l}\text { Time to fever resolution } \\
\text { Hospital mortality } \\
\text { Time to resolution of organ } \\
\text { dysfunction (renal failure, } \\
\text { hepatic dysfunction, and } \\
\text { thrombocytopenia) }\end{array}$ \\
\hline
\end{tabular}


Table 1. Cont.

\begin{tabular}{|c|c|c|c|c|c|c|}
\hline $\begin{array}{c}\text { Author (Year) and } \\
\text { Reference }\end{array}$ & Treatment/Prophylaxis & Type of Study & Patients & Treatment Groups & $n$ & Outcomes \\
\hline $\begin{array}{c}\text { Suputtamongkol Y (2004) } \\
{[21]}\end{array}$ & Treatment & Randomized, open trial & $\begin{array}{l}\text { Patients with suspected } \\
\text { severe leptospirosis in some } \\
\text { hospitals in Thailand, } \\
\text { whom had isolated } \\
\text { leptospires from blood or a } \\
4^{\prime} \text { fold or greater increase in } \\
\text { the antibody titer or a single } \\
\text { or stable antibody titer of } \\
\geq 1: 400 .\end{array}$ & $\begin{array}{l}\text { Penicillin G } 1.5 \mathrm{MU} / 6 \mathrm{~h} \\
\text { versus Cefotaxime } 1 \mathrm{~g} / 6 \mathrm{~h} \\
\text { versus Doxycycline } 200 \mathrm{mg} \\
\text { first dose followed by } 100 \\
\mathrm{mg} / 12 \mathrm{~h} \text { iv. } 7 \text { days }\end{array}$ & $\begin{array}{l}181 \text { vs. } 172 \text { vs. } 187 \\
\text { (87 vs. } 81 \text { vs. } 88 \text { with } \\
\text { confirmed leptospirosis) }\end{array}$ & $\begin{array}{c}\text { Mortality; } \\
\text { Time to defervescence; } \\
\text { Duration of hospitalization }\end{array}$ \\
\hline Phimda K (2007) [22] & Treatment & Randomized trial & $\begin{array}{l}\text { Patients with suspected } \\
\text { Leptospirosis and scrub } \\
\text { typhus in Thailand } \\
\text { Leptospirosis: isolation } \\
\text { from blood or positive } \\
\text { serologic test (fourfold or } \\
\text { greater rise in antibody titer } \\
\text { or at least 1:400 on a single } \\
\text { specimen). }\end{array}$ & $\begin{array}{l}\text { Doxycycline } 200 \mathrm{mg} \text { the first } \\
\text { dose followed by } 100 \\
\mathrm{mg} / 12 \text { horas seven days } \\
\text { versus } \\
\text { Azithromycin } 1 \mathrm{~g} \text { initially } \\
\text { followed by } 500 \mathrm{mg} \text { once } \\
\text { daily for } 2 \text { days. }\end{array}$ & 34:35 (only leptospirosis) & $\begin{array}{l}\text { Time of fever clearance; } \\
\% \text { of afebrile patients at } 48 \mathrm{~h} \\
\text { post-treatment. }\end{array}$ \\
\hline Durich J (1956) [23] & Chemoprophylaxis & Probably non randomized ${ }^{\dagger}$ & $\begin{array}{l}\text { Rice reapers in the province } \\
\text { of Valencia (Spain) }\end{array}$ & $\begin{array}{c}\text { Penicillin procain } 100,000 \\
\mathrm{U} / 12 \mathrm{~h} \text { vo (for } 100 \text { reapers) } \\
\text { or dipenicillin } \\
\mathrm{N}, \mathrm{N}^{\prime} \text { dibenciletilendiamine } \\
100000 \mathrm{U} / 12 \mathrm{~h} \text { (for } 50 \\
\text { reapers) versus control. }\end{array}$ & 150 vs. 150 & New cases of leptospirosis. \\
\hline Takafuji ET (1984) [24] & Chemoprophylaxis & $\begin{array}{l}\text { Double-blind, } \\
\text { placebo-controlled trial }\end{array}$ & $\begin{array}{c}\text { Healthy volunteers at } \\
\text { Jungle Operations Training } \\
\text { Center (Panama). }\end{array}$ & $\begin{array}{l}\text { Doxycycline } 200 \mathrm{mg} \text { weekly } \\
\text { vs. placebo. }\end{array}$ & $469: 471$ & New cases of leptospirosis. \\
\hline Gonsalez CR (1998) [25] & Chemoprophylaxis & $\begin{array}{l}\text { Double-blind, randomized, } \\
\text { placebo-controlled trial }\end{array}$ & $\begin{array}{l}\text { Residents of a small } \\
\text { community in Sao Paulo } \\
\text { region, an area at high risk } \\
\text { for flooding. }\end{array}$ & $\begin{array}{l}\text { Doxycycline } 200 \mathrm{mg} \text { single } \\
\text { dose vs. placebo. }\end{array}$ & 40 vs. 42 & $\begin{array}{l}\text { New confirmed cases } \\
\text { (symptomatic). } \\
\text { Seropositivity }\end{array}$ \\
\hline Sehgal SC (2000) [26] & Chemoprophylaxis & Randomized controlled-trial & $\begin{array}{l}\text { Healthy persons ( }>10 \text { years } \\
\text { old) residing in Diglipur } \\
\text { (North Andaman), India. }\end{array}$ & $\begin{array}{c}\text { Doxycycline } 200 / \text { week } \\
\text { versus placebo (12 weeks). }\end{array}$ & 386 vs. 396 & $\begin{array}{c}\text { New cases of leptospirosis. } \\
\text { Seropositivity }\end{array}$ \\
\hline
\end{tabular}


Table 1. Cont.

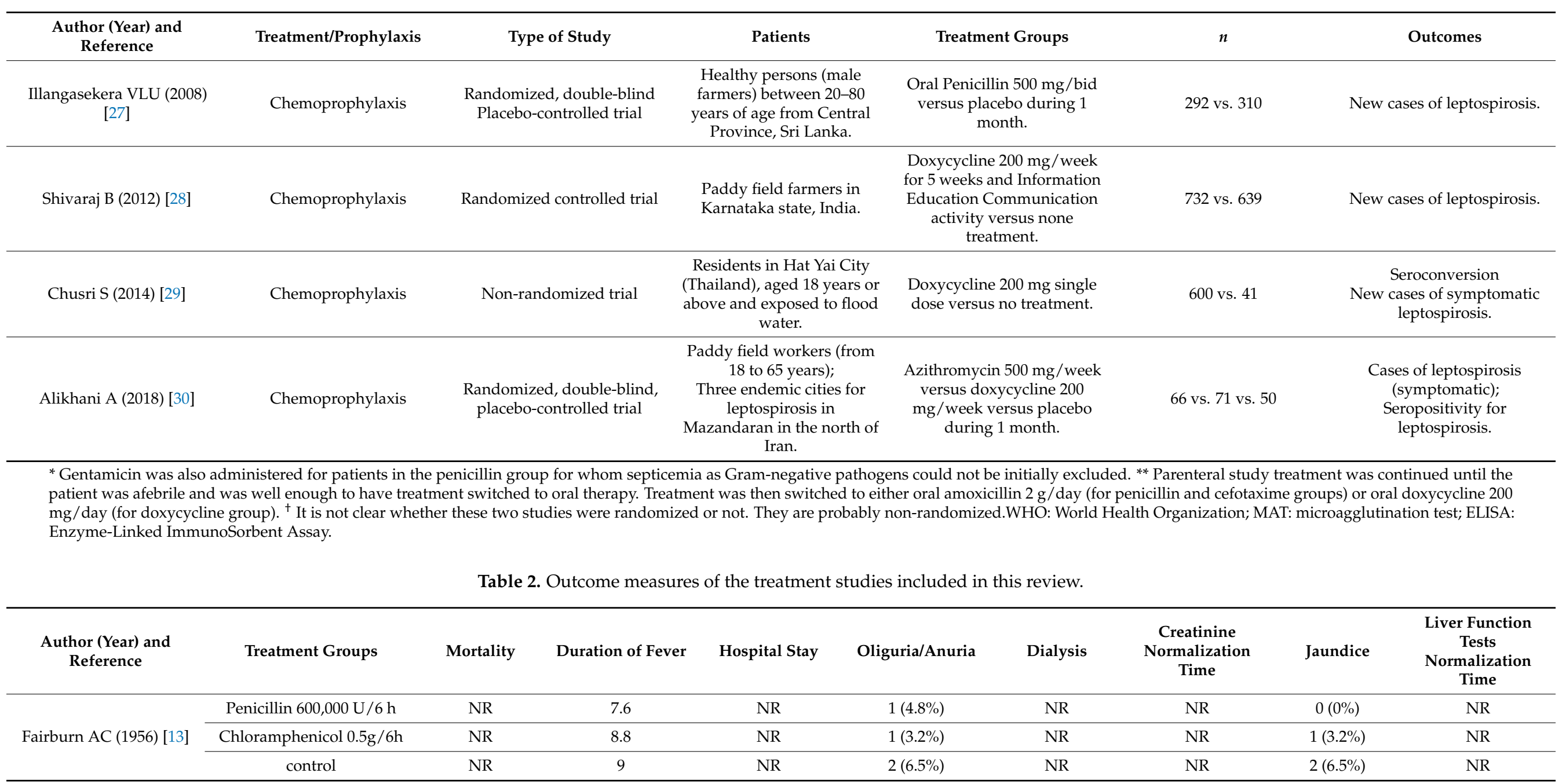


Table 2. Cont.

\begin{tabular}{|c|c|c|c|c|c|c|c|c|c|}
\hline $\begin{array}{l}\text { Author (Year) and } \\
\text { Reference }\end{array}$ & Treatment Groups & Mortality & Duration of Fever & Hospital Stay & Oliguria/Anuria & Dialysis & $\begin{array}{c}\text { Creatinine } \\
\text { Normalization } \\
\text { Time }\end{array}$ & Jaundice & $\begin{array}{c}\text { Liver Function } \\
\text { Tests } \\
\text { Normalization } \\
\text { Time }\end{array}$ \\
\hline \multirow[t]{2}{*}{ Ross Russell (1958) [14] } & $\begin{array}{c}\text { Oxytetracycline } 1.5 \mathrm{~g} \\
\text { followed by } 0.5 \mathrm{~g} / 6 \mathrm{~h} \text { for at } \\
\text { least } 5 \text { days }\end{array}$ & NR & 6.4 & NR & NR & NR & NR & $6(22.2 \%)$ & NR \\
\hline & placebo & NR & 9.4 & NR & NR & NR & NR & $5(20 \%)$ & NR \\
\hline \multirow{2}{*}{ McClain (1984) [15] } & $\begin{array}{l}\text { Doxiciclina } 100 \mathrm{mg} \text { cada } 12 \\
\text { horas durante } 7 \text { días }\end{array}$ & NR & $3.7 \pm 0.3$ & NR & NR & NR & NR & NR & NR \\
\hline & placebo & NR & $5.4 \pm 0.3$ & NR & NR & NR & NR & NR & NR \\
\hline \multirow{2}{*}{ Watt G (1988) [16] } & $\begin{array}{c}\text { Penicillin G } 1.5 \mathrm{MU} / 6 \mathrm{~h} \text { for } \\
7 \text { days }\end{array}$ & $0 / 23$ & $4.7 \pm 4.19$ & NR & NR & $0(0 \%)$ & $2.7 \pm 1.9$ & NR & NR \\
\hline & placebo & $0 / 19$ & $11.6 \pm 8.34$ & NR & NR & $0(0 \%)$ & $8.3 \pm 8.46$ & NR & NR \\
\hline \multirow{2}{*}{ Edwards CN (1988) [17] } & $\begin{array}{c}\text { Penicillin } 2 \mathrm{MU} / 6 \mathrm{~h} \text { iv } 5 \\
\text { days }\end{array}$ & $1 / 38$ & $6.9 \pm 3.8$ & NR & NR & NR & NR & NR & $5.7 \pm 3$ \\
\hline & placebo & $3 / 41$ & $6.6 \pm 3.2$ & NR & NR & NR & NR & NR & $5.65 \pm 3.7$ \\
\hline \multirow{2}{*}{ Daher EF (2000) [18] } & $\begin{array}{l}\text { Penicillin } 6 \mathrm{MU} / \text { day iv for } \\
8 \text { days }\end{array}$ & $1 / 16$ & $3 \pm 4$ & $12 \pm 6$ & $3(19 \%)$ & $8(50 \%)$ & $10 \pm 6$ & NR & $8 \pm 3$ \\
\hline & no antibiotics & $0 / 19$ & $2 \pm 3$ & $11 \pm 5$ & $1(5 \%)$ & $10(52 \%)$ & $9 \pm 6$ & NR & $8 \pm 3$ \\
\hline \multirow{2}{*}{ Costa E (2003) [19] } & $\begin{array}{l}\text { Penicillin } 6 \mathrm{mU} / \text { day (1 } \\
\mathrm{mU} / 4 \mathrm{~h} \text { ) for seven days }\end{array}$ & $15 / 125$ & NR & $8.9 \pm 3.9$ & NR & $35(28 \%)$ & NR & NR & NR \\
\hline & control & $8 / 128$ & NR & $8.8 \pm 3.6$ & NR & $23(18 \%)$ & NR & NR & NR \\
\hline \multirow[b]{2}{*}{ Panaphut T (2003) [20] } & $\begin{array}{c}\text { penicillin } \mathrm{G} 1.5 \mathrm{MU} / 6 \mathrm{~h} \text { for } \\
7 \text { days }\end{array}$ & $5 / 86$ & $3 *$ & NR & NR & NR & NR & NR & NR \\
\hline & $\begin{array}{l}\text { Ceftriaxone } 1 \mathrm{~g} / \text { day for } 7 \\
\text { days }\end{array}$ & $5 / 87$ & $3 *$ & NR & NR & NR & NR & NR & NR \\
\hline
\end{tabular}


Table 2. Cont

\begin{tabular}{|c|c|c|c|c|c|c|c|c|c|}
\hline $\begin{array}{l}\text { Author (Year) and } \\
\text { Reference }\end{array}$ & Treatment Groups & Mortality & Duration of Fever & Hospital Stay & Oliguria/Anuria & Dialysis & $\begin{array}{c}\text { Creatinine } \\
\text { Normalization } \\
\text { Time }\end{array}$ & Jaundice & $\begin{array}{l}\text { Liver Function } \\
\text { Tests } \\
\text { Normalization } \\
\text { Time }\end{array}$ \\
\hline \multirow{3}{*}{$\begin{array}{l}\text { Suputtamongkol Y } \\
\text { (2004) [21] }\end{array}$} & $\begin{array}{c}\text { Penicillin G } 1.5 \text { MU / } 6 \text { h } 7 \\
\text { days }\end{array}$ & $2 / 87$ & $72(12-240)$ & $6(2-21)$ & NR & NR & NR & NR & NR \\
\hline & Cefotaxime $1 \mathrm{~g} / 6 \mathrm{~h} 7$ days & $0 / 88$ & $60(8-192)$ & $5.5(3-37)$ & NR & NR & NR & NR & NR \\
\hline & $\begin{array}{l}\text { Doxycycline } 200 \mathrm{mg} \text { first } \\
\text { dose followed by } 100 \\
\mathrm{mg} / 12 \mathrm{~h} \text { iv. } 7 \text { days. }\end{array}$ & $2 / 81$ & $72(12-264)$ & $5(2-28)$ & NR & NR & NR & NR & NR \\
\hline \multirow{2}{*}{ Phimda K (2007) [22] } & $\begin{array}{l}\text { Doxycycline } 200 \mathrm{mg} \text { the } \\
\text { first dose followed by } 100 \\
\mathrm{mg} / 12 \text { horas seven days }\end{array}$ & NR & $45 \mathrm{~h}(8-118 \mathrm{~h})$ & NR & NR & NR & NR & NR & NR \\
\hline & $\begin{array}{l}\text { Azithromycin } 1 \mathrm{~g} \text { initially } \\
\text { followed by } 500 \mathrm{mg} \text { once } \\
\text { daily for } 2 \text { days. }\end{array}$ & NR & $40 \mathrm{~h}(8-136 \mathrm{~h})$ & NR & NR & NR & NR & NR & NR \\
\hline
\end{tabular}

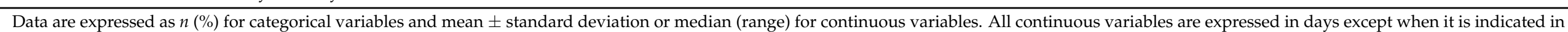
hours (h). ${ }^{*}$ Median. NR: not reported. 
Outcome measures of the treatment studies included in this review are in Table 2. The meta-analysis showed no effect of penicillin treatment on mortality compared to placebo or no antibiotic treatment (OR 1.60; 95\% CI 0.59-4.31; $p=0.36$ ) (Figure 3). Some trials even showed lower mortality in those who received a placebo over penicillin treatment $[18,19]$. It has not been proven either differences between penicillin and other antibiotic treatments such as cephalosporins (OR 1.29; 95\% CI 0.40-4.19; $p=0.67$ ) or doxycycline (OR 0.93; 95\% CI 0.13-6.76; $p=0.94$ ) (Figure 3). Neither effect of penicillin has been demonstrated on the time of defervescence (MD -1.35; 95\% CI $(-4.82)-2.12 ; p=0.45)$ or on hospital stay (MD 0.15; 95\% CI $(-0.75)-1.06 ; p=0.74)$. (Figure 3b,c). Only Watt's study seemed to show a difference in reducing the duration of fever [16]. Besides, the data also did not demonstrate any effectiveness for the use of penicillin on the incidence of oliguria/anuria, the need for dialysis treatment, or on time to creatinine normalization (Figure $4 a, b$ ). There was also no benefit in the incidence of jaundice or in the liver function normalization time (Figure 5a,b).

Eight trials assessed prophylactic treatment against leptospirosis with different strategies: four of them with $200 \mathrm{mg}$ of doxycycline weekly [24,26,28,30], two with daily oral penicillin $[23,27]$, another two with a single dose of doxycycline at the time of exposure to flood water $[25,29]$. One of them also compared doxycycline prophylaxis with a weekly azithromycin regimen [30]. Among all these trials, they comprise 4905 patients. The results of the administration of a weekly dose of $200 \mathrm{mg}$ of doxycycline versus placebo or no antibiotics do not show statistical significance (OR 0.20; 95\% CI 0.02-1.87; $p=0.16$ ) (Figure 6). There was enormous heterogeneity among the studies. Two of them showed a clear benefit of doxycycline [24,28], but the most recent trial result did not show this benefit [30]. However, this study showed a lower percentage of new IgG seropositivity in those patients who received chemoprophylaxis than in the placebo group (Table 3) [30]. The administration of a single dose of doxycycline at times of exposure reached statistical significance that favors doxycycline (OR 0.23; 95\% CI 0.07-0.77; $p=0.02$ ) (Figure 6). However, one of the studies included in this latter comparison was not randomized [29]. None of the rest of the comparisons have shown statistical significance in favor of intervention with antibiotics in the prophylaxis of clinical leptospirosis, nor the administration of oral penicillin (OR 0.17; 95\% CI 0.02-1.44; $p=0.10$ ), nor a regimen that includes azithromycin (OR 0.75; $95 \%$ CI $0.10-5.52 ; p=0.78$ ) (Figure 6), although there seems to be a tendency to have fewer cases of leptospirosis in the groups that have received chemoprophylaxis regimens. 
(a) Mortality

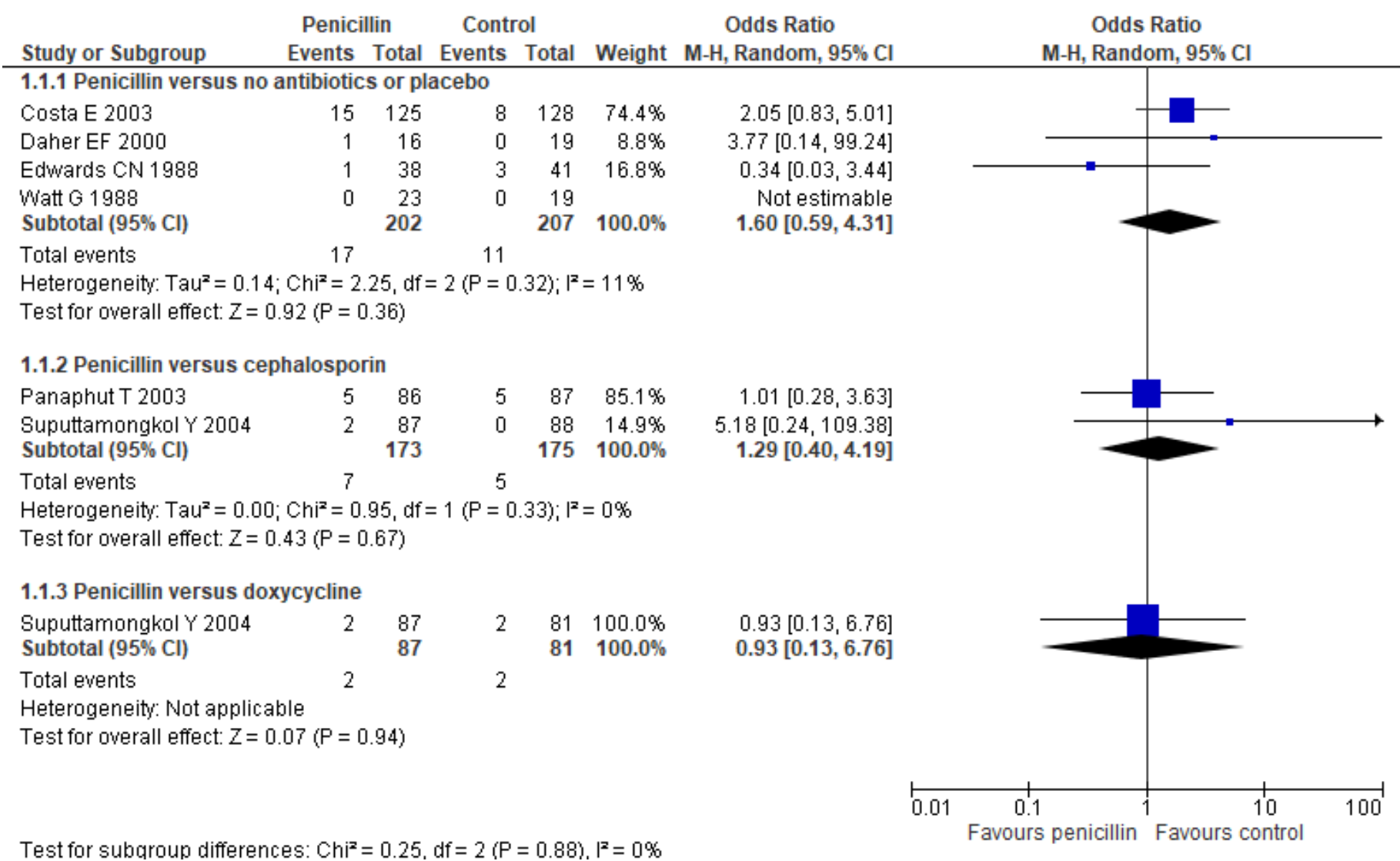

(b) Time to defervescence.

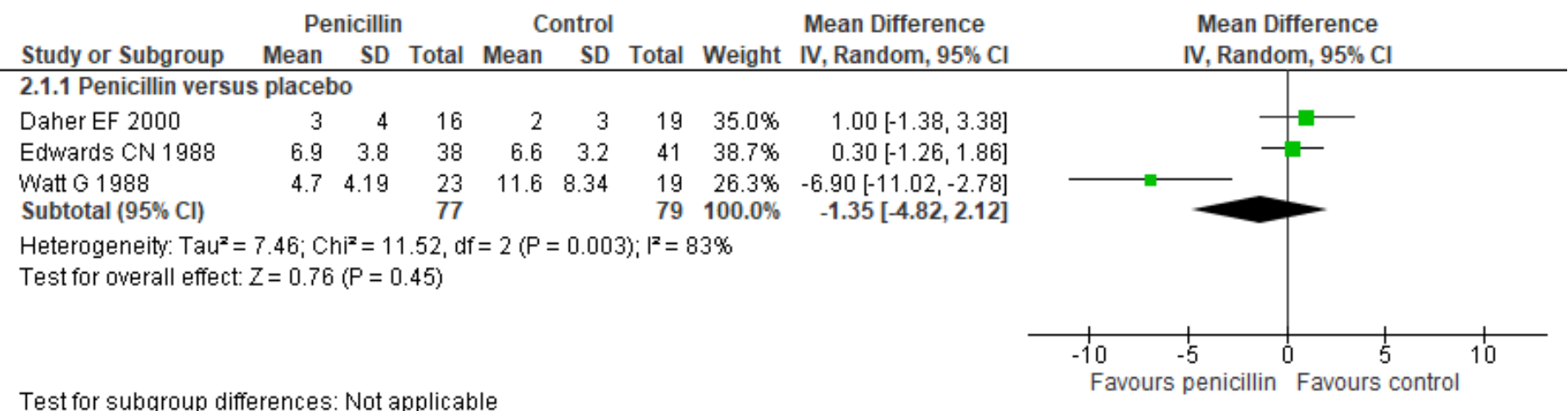

(c) Hospital stay.

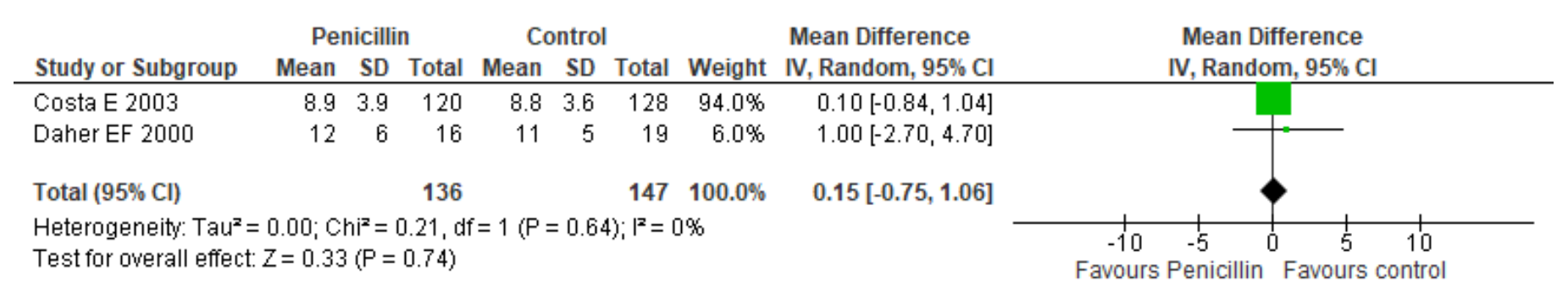

Figure 3. Forest plots on the comparisons on antibiotic treatment efficacy: mortality (a), time to defervescence (b), and length of hospital stay (c). The figure shows the results in treatment and control groups for each study. The sizes of the colored boxes are relative to the study weight. The meta-analysis for each comparison is shown as a diamond. 
(a) Patients with oliguria/anuria or with the need of dialysis.

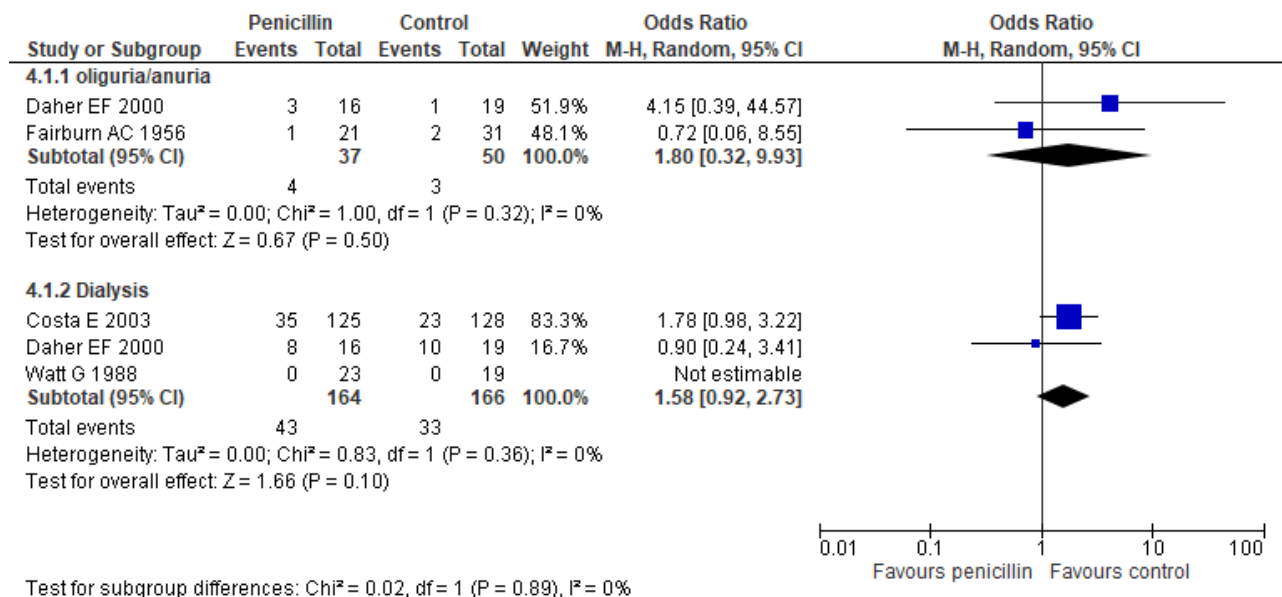

(b) Creatinine normalization time.

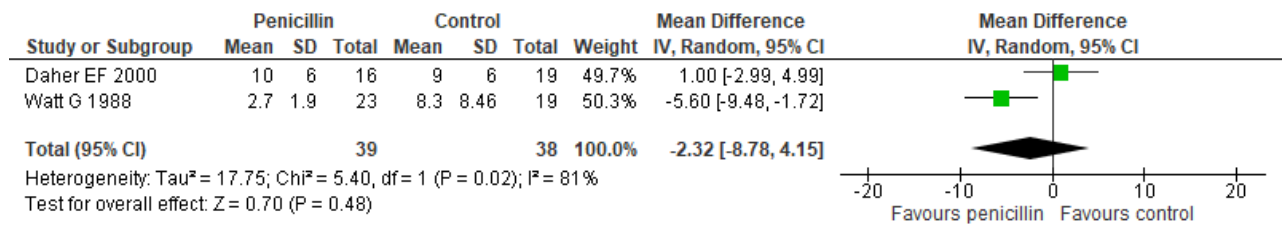

Figure 4. Forest plots of the comparisons on antibiotic treatment effect on renal dysfunction. (a) Patients with oliguria/anuria or need of dialysis and $(\mathbf{b})$ creatinine normalization time. The figure shows the results in treatment and control groups for each study. The sizes of colored boxes are relative to the study weight. The meta-analysis for each comparison is shown as a diamond.

(a) Jaundice incidence.

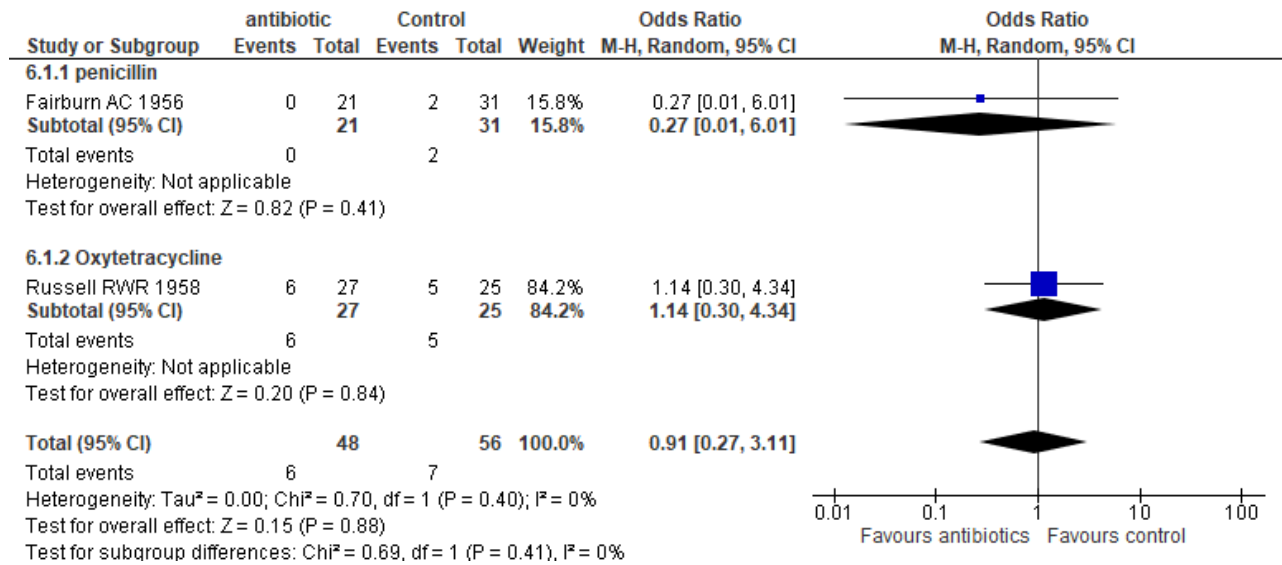

(b) Liver function normalization time.

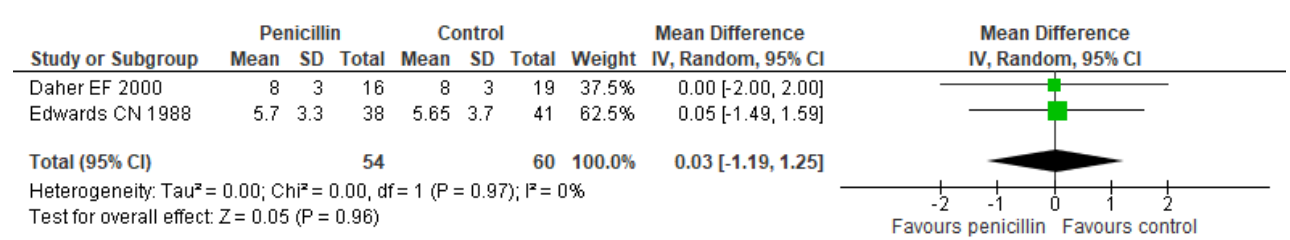

Figure 5. Forest plots of the comparisons on the effect of antibiotics on liver dysfunction. The figure shows the results in treatment and control groups for each study. (a) Patients with jaundice (b) liver function normalization time.The sizes of colored boxes are relative to the study weight. The meta-analysis for each comparison is shown as a diamond. 


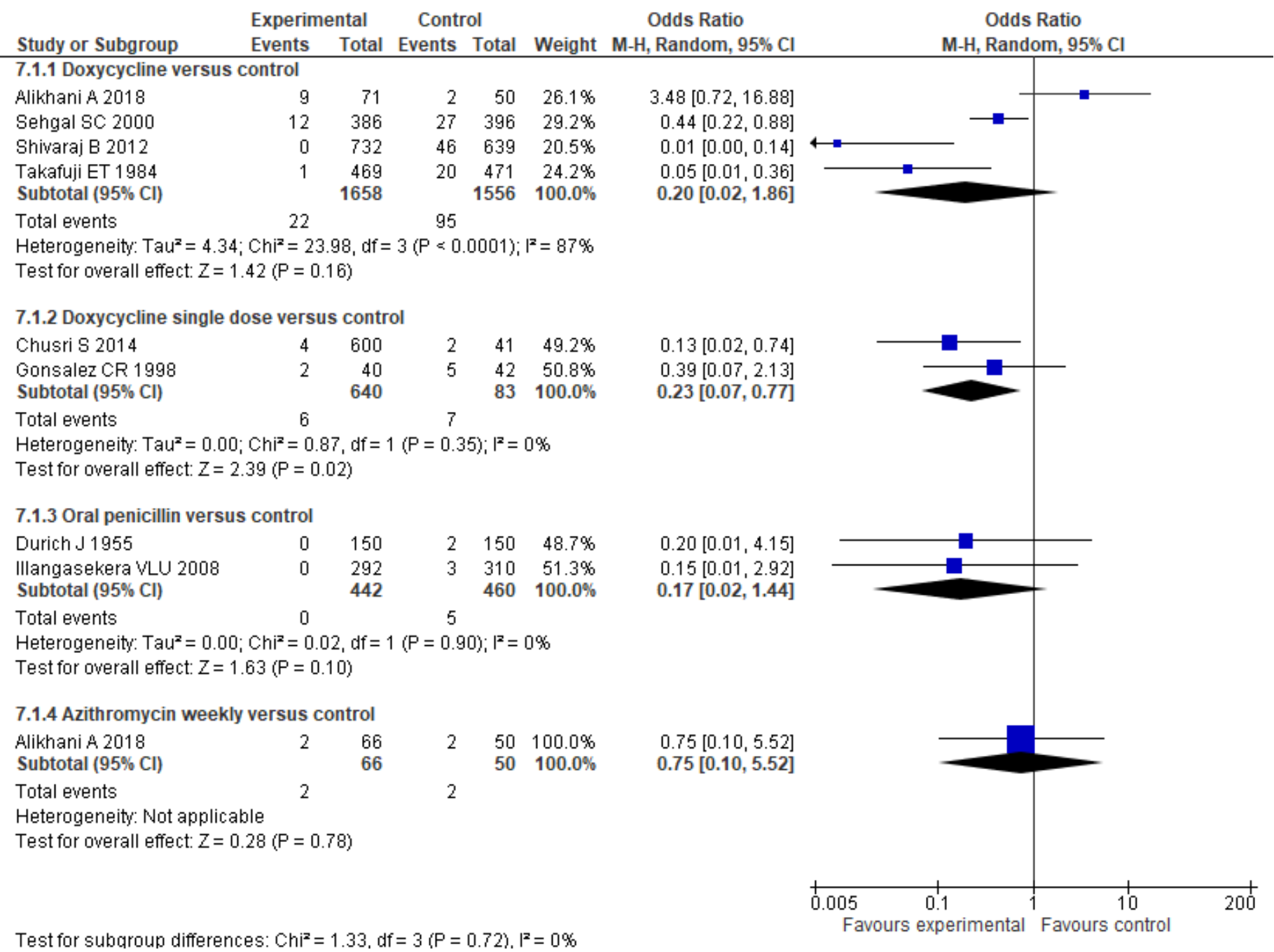

Figure 6. Forest plots of the comparisons on chemoprophylaxis efficacy: number of new symptomatic cases. The figure shows the number of participants and events in treatment and control groups for each study. The sizes of colored boxes are relative to the study weight. The meta-analysis for each comparison is shown as a diamond.

Table 3. Outcome measures of the chemoprophylaxis studies included in this review.

\begin{tabular}{cccc}
\hline Author (Year) & Treatment Groups & New Symptomatic Cases & Seroconversion \\
\hline \multirow{2}{*}{ Durich J (1956) [23] } & $\begin{array}{c}\text { Penicillin procain 100,000 } \\
\text { U/12 h vo or dipenicillin } \\
\text { N,N'dibenciletilendiamine }\end{array}$ & $0 / 150(0 \%)$ & NR \\
\cline { 2 - 4 } & Control & $2 / 150(1.3 \%)$ & NR \\
Takafuji ET (1984) [24] & $\begin{array}{c}\text { Doxycycline 200 mg } \\
\text { semanales (3 weeks aprox) }\end{array}$ & $1 / 469(0.2 \%)$ & NR \\
\cline { 2 - 4 } & placebo & $20 / 471(4.2 \%)$ & IgM: $13 / 40(32.5 \%)$ \\
\hline Gonsalez CR (1998) [25] & $\begin{array}{c}\text { Doxycycline 200 mg single } \\
\text { dose }\end{array}$ & $2 / 40(5 \%)$ & IgM: $11 / 42(26.2 \%)$ \\
\cline { 2 - 4 } & placebo & $5 / 42(11.9 \%)$ & $112 / 386(29 \%)$ \\
Sehgal SC (2000) [26] & Doxycycline 200/week (12 & $12 / 386(3.1 \%)$ & $101 / 396(25.5 \%)$ \\
\cline { 2 - 4 }
\end{tabular}


Table 3. Cont.

\begin{tabular}{|c|c|c|c|}
\hline Author (Year) & Treatment Groups & New Symptomatic Cases & Seroconversion \\
\hline \multirow{2}{*}{ Illangasekera VLU (2008) [27] } & $\begin{array}{l}\text { Oral Penicillin } 500 \mathrm{mg} / \mathrm{bid} \text { for } \\
\text { one month }\end{array}$ & 0/292 (0\%) & NR \\
\hline & placebo & $3 / 310(1 \%)$ & NR \\
\hline \multirow{2}{*}{ Shivaraj B (2012) [28] } & $\begin{array}{l}\text { Doxycycline } 200 \mathrm{mg} / \text { week for } \\
5 \text { weeks and IEC }\end{array}$ & $0 / 732(0 \%)$ & NR \\
\hline & No treatment & $46 * / 639(7.29 \%)$ & NR \\
\hline \multirow{2}{*}{ Chusri S (2014) [29] } & $\begin{array}{l}\text { Doxycycline } 200 \mathrm{mg} \text { single } \\
\text { dose }\end{array}$ & $4 / 600(0.7 \%)$ & $17 / 600(2.8 \%)$ \\
\hline & No treatment & $2 / 41(4.9 \%)$ & $5 / 41(12.2 \%)$ \\
\hline \multirow{3}{*}{ Alikhani A (2018) [30] } & Azithromycin $500 \mathrm{mg} /$ week & $2 / 66(3 \%)$ & $\begin{array}{l}\text { IgM: } 2 / 66(3 \%) \\
\text { IgG: } 5 / 66(7.6 \%)\end{array}$ \\
\hline & doxycycline $200 \mathrm{mg}$ /week & $9 / 71(12.6 \%)$ & $\begin{array}{l}\text { IgM: } 9 / 71(12.6 \%) \\
\text { IgG: } 8 / 71(11.3 \%)\end{array}$ \\
\hline & placebo & $2 / 50(4 \%)$ & $\begin{array}{c}\text { IgM: } 2 / 50(4 \%) \\
\text { IgG: } 12 / 50(24 \%)\end{array}$ \\
\hline
\end{tabular}

Data are expressed as the number of new symptomatic cases/total patients included (\%), or the number of new positive antibody cases/total patients included (\%). NR: not reported. * This study gives percentage and no the number of new symptomatic cases. 46 was deduced from the data of this study.

\section{Discussion}

This study provides a meta-analysis on the use of antibiotics in leptospirosis both for treatment and for chemoprophylaxis. The most remarkable thing is that there is a lack of good quality studies on the efficacy of antibiotics at various stages of the disease, and no significant treatment effect has been detected. On the other hand, although most chemoprophylaxis studies show fewer cases of leptospirosis with chemoprophylaxis, they do not reach statistical significance. Therefore, large and good quality studies are needed that consider how leptospirosis was diagnosed and at what stage of the disease treatment is given to detect an effect of treatment.

Another notable fact is the absence of new clinical trials with antibiotics in recent years. The last published trial on treatment was published in 2007 [22], and since that year, no new clinical trials have been published. In addition, several trials were published more than 30 years ago, with a small number of patients and with a non-rigorous methodology regarding the use of placebo or the randomization of patients. A similar result has already been reported by Charan J et al. and Brett-Major DM et al. [31,32]. These authors showed the absence of significant differences between penicillin and placebo in terms of mortality, duration of fever, and renal impairment in leptospirosis. Our work also documents the absence of significant effects in terms of the duration of liver and kidney dysfunction. Besides, our analysis includes information from chemoprophylaxis studies, which gives a complete view of the evidence on the use of antibiotics in this infectious disease. Welldesigned studies should be done on the effect of antibiotics at different stages of the disease. Most human diagnostics are serological. MAT has been considered the gold standard for diagnosis [8]. This leads to antibiotics are often started when the immune response is clearing the leptospires. Despite its difficulty, this data justifies studies to describe the effect of antibiotics at different times: exposure, leptospiraemic, and immune phases. The paucity of recent, well-designed trials confirms the idea that leptospirosis remains a neglected tropical disease.

In recent years, trials have been carried out to verify the effect of non-antibiotic treatments such as corticosteroids [33,34] or other drugs [35] in severe leptospirosis, although without a clear result at the moment. On the other hand, although other authors have 
advocated testing other antibiotics such as quinolones [36], no trials have been conducted for this purpose.

Regarding prophylaxis, the results did not show statistical significance in most comparisons. The antibiotics tested for prophylaxis are the same as those tested for treatment, although some prophylaxis trials being more recent than those ones carried out for treatment. On the other hand, the design of the trials is very uneven in the time of prophylaxis (single or weekly dose). Only when a single dose of doxycycline was used at the moment of floodwater exposition, chemoprophylaxis showed benefit with statistical significance. However, this effect is based on the results of a non-randomized study [29]. However, in most studies, there seems to be a tendency to have fewer cases of leptospirosis in the groups with chemoprophylaxis regimens. Only the Alikhani study [30] shows more cases of clinical leptospirosis in the doxycycline chemoprophylaxis group than in the control group. Nevertheless, in this study, there are fewer cases of new IgG seropositivity patients in the doxycycline group. Probably large studies are needed to clarify the role and efficacy of chemoprophylaxis in human leptospirosis.

Many unanswered questions remain regarding the treatment and prophylaxis of leptospirosis. It has been suggested that early treatment could be more effective, but it is not known from what day in the clinical course (if there is one) the treatment becomes less effective. It is also not known whether other antibiotics such as quinolones or aminoglycosides may play a role. Only randomized trials with a sufficient number of patients can answer these questions. Meanwhile, in the absence of better evidence, treatment with penicillin and doxycycline continues to be recommended in medical texts, but there is no substantial progress on this issue in the last 30 years. This fact confirms that leptospirosis continues to be a neglected disease.

Author Contributions: Conceptualization, M.G.P., J.S.G.d.P., and J.J.B.S.; methodology, M.G.P. and J.S.G.d.P.; formal analysis, J.S.G.d.P.; investigation, J.S.G.d.P. and M.G.P.; resources, M.G.P., J.S.G.d.P., J.J.B.S.; data curation, M.G.P. and J.S.G.d.P.; writing—original draft preparation, J.S.G.d.P.; writingreview and editing, F.M.R., J.J.B.S., J.C.S.L., E.M.A., M.G.P., and J.S.G.d.P.; visualization, F.M.R., J.J.B.S., J.C.S.L., E.M.A., M.G.P., and J.S.G.d.P.; supervision, J.S.G.d.P. and J.J.B.S. All authors have read and agreed to the published version of the manuscript.

Funding: This research received no external funding.

Conflicts of Interest: The authors declare no conflict of interest.

\section{References}

1. Cagliero, J.; Villanueva, S.Y.A.M.; Matsui, M. Leptospirosis pathophysiology: Into the storm of cytokines. Front. Cell Infect Microbiol. 2018, 8, 204. [CrossRef]

2. Levett, P.N. Leptospirosis. Clin. Microbiol. Rev. 2001, 14, 296-326. [CrossRef] [PubMed]

3. Haake, D.A.; Levett, P.N. Leptospirosis in humans. Curr. Top Microbiol. Immunol. 2015, 387, 65-97. [CrossRef]

4. De Brito, T.; Silva, A.M.G.D.; Abreu, P.A.E. Pathology and pathogenesis of human leptospirosis: A commented review. Rev. Inst. Med. Trop. Sao Paulo 2018, 60, e23. [CrossRef]

5. Le Turnier, P.; Epelboin, L. Mise au point sur la leptospirose [Update on leptospirosis]. Rev. Med. Interne. 2019, 40, 306-312. [CrossRef]

6. Bharti, A.R.; Nally, J.E.; Ricaldi, J.N.; Matthias, M.A.; Diaz, M.M.; Lovett, M.A.; Levett, P.N.; Gilman, R.H.; Willig, M.R.; Gotuzzo, E.; et al. Leptospirosis: A zoonotic disease of global importance. Lancet Infect. Dis. 2003, 3, 757-771. [CrossRef]

7. Soo, Z.M.P.; Khan, N.A.; Siddiqui, R. Leptospirosis: Increasing importance in developing countries. Acta Trop. 2020, 201, 105183. [CrossRef] [PubMed]

8. Samrot, A.V.; Sean, T.C.; Bhavya, K.S.; Sahithya, C.S.; Chan-Drasekaran, S.; Palanisamy, R.; Robinson, E.R.; Subbiah, S.K.; Mok, P.L. Leptospiral infection, pathogenesis and its diagnosis-A review. Pathogens 2021, 10, 145. [CrossRef] [PubMed]

9. Goarant, C. Leptospirosis: Risk factors and management challenges in developing countries. Res. Rep. Trop. Med. 2016, 7, 49-62. [CrossRef]

10. Costa, F.; Hagan, J.E.; Calcagno, J.; Kane, M.; Torgerson, P.; Martinez-Silveira, M.S.; Stein, C.; Abela-Ridder, B.; Ko, A.I. Global morbidity and mortality of leptospirosis: A systematic review. PLoS Negl. Trop. Dis. 2015, 9, e0003898. [CrossRef]

11. Griffith, M.E.; Hospenthal, D.R.; Murray, C.K. Antimicrobial therapy of leptospirosis. Curr. Opin. Infect. Dis. 2006, 19, 533-537. [CrossRef] 
12. Higgins, J.; Thomas, J. Cochrane Handbook for Systematic Reviews of Interventions; John Wiley \& Sons: Hoboken, NJ, USA, 2019; Version 6.

13. Fairburn, A.C.; Semple, S.J. Chloramphenicol and penicillin in the treatment of leptospirosis among British troops in Malaya. Lancet 1956, 270, 13-16. [CrossRef]

14. Russell, R.W. Treatment of leptospirosis with oxytetracycline. Lancet 1958, 2, 1143-1145. [CrossRef]

15. McClain, J.B.; Ballou, W.R.; Harrison, S.M.; Steinweg, D.L. Doxycycline therapy for leptospirosis. Ann. Intern. Med. 1984, 100, 696-698. [CrossRef] [PubMed]

16. Watt, G.; Padre, L.P.; Tuazon, M.L.; Calubaquib, C.; Santiago, E.; Ranoa, C.P.; Laughlin, L.W. Placebo-controlled trial of intravenous penicillin for severe and late leptospirosis. Lancet 1988, 1, 433-435. [CrossRef]

17. Edwards, C.N.; Nicholson, G.D.; Hassell, T.A.; Everard, C.O.; Callender, J. Penicillin therapy in icteric leptospirosis. Am. J. Trop . Med. Hyg. 1988, 39, 388-390. [CrossRef]

18. Daher, E.F.; Nogueira, C.B. Evaluation of penicillin therapy in patients with leptospirosis and acute renal failure. Rev. Inst. Med. Trop. Sao Paulo 2000, 42, 327-332. [CrossRef]

19. Costa, E.; Lopes, A.A.; Sacramento, E.; Costa, Y.A.; Matos, E.D.; Lopes, M.B.; Bina, J.C. Penicillin at the late stage of leptospirosis: A randomized controlled trial. Rev. Inst. Med. Trop. Sao Paulo 2003, 45, 141-145. [CrossRef]

20. Panaphut, T.; Domrongkitchaiporn, S.; Vibhagool, A.; Thinkamrop, B.; Susaengrat, W. Ceftriaxone compared with sodium penicillin $\mathrm{g}$ for treatment of severe leptospirosis. Clin. Infect. Dis. 2003, 36, 1507-1513. [CrossRef]

21. Suputtamongkol, Y.; Niwattayakul, K.; Suttinont, C.; Losuwanaluk, K.; Limpaiboon, R.; Chierakul, W.; Wuthiekanun, V.; Triengrim, S.; Chenchittikul, M.; White, N.J. An open, randomized, controlled trial of penicillin, doxycycline, and cefotaxime for patients with severe leptospirosis. Clin. Infect. Dis. 2004, 39, 1417-1424. [CrossRef]

22. Phimda, K.; Hoontrakul, S.; Suttinont, C.; Chareonwat, S.; Losuwanaluk, K.; Chueasuwanchai, S.; Chierakul, W.; Suwancharoen, D.; Silpasakorn, S.; Saisongkorh, W.; et al. Doxycycline versus azithromycin for treatment of leptospirosis and scrub typhus. Antimicrob. Agents Chemother. 2007, 51, 3259-3263. [CrossRef] [PubMed]

23. Durich, J.; Pumarola, A. La endemia de leptospirosis en la provincia de Valencia. ensayo de métodos profilácticos (1954) [Endemic aspects of leptospirosis in the province of Valencia; trial of preventive methods (1954)]. Med. Esp. 1955, 34, 83-90. [PubMed]

24. Takafuji, E.T.; Kirkpatrick, J.W.; Miller, R.N.; Karwacki, J.J.; Kelley, P.W.; Gray, M.R.; McNeill, K.M.; Timboe, H.L.; Kane, R.E.; Sanchez, J.L. An efficacy trial of doxycycline chemoprophylaxis against leptospirosis. N. Engl. J. Med. 1984, 310, 497-500. [CrossRef]

25. Gonsalez, C.R.; Casseb, J.; Monteiro, F.G.; Paula-Neto, J.B.; Fernandez, R.B.; Silva, M.V.; Camargo, E.D.; Mairinque, J.M.; Tavares, L.C. Use of doxycycline for leptospirosis after high-risk exposure in São Paulo, Brazil. Rev. Inst. Med. Trop. Sao Paulo 1998, 40, 59-61. [CrossRef] [PubMed]

26. Sehgal, S.C.; Sugunan, A.P.; Murhekar, M.V.; Sharma, S.; Vijayachari, P. Randomized controlled trial of doxycycline prophylaxis against leptospirosis in an endemic area. Int. J. Antimicrob. Agents 2000, 13, 249-255. [CrossRef]

27. Illangasekera, V.L.; Kularatne, S.A.; Kumarasiri, P.V.; Pussepitiya, D.; Premaratne, M.D. Is oral penicillin an effective chemoprophylaxis against leptospirosis? A placebo controlled field study in the Kandy District, Sri Lanka. Southeast Asian J. Trop. Med. Public Health 2008, 39, 882-884.

28. Shivaraj, B.; Ts, R.; Anithraj, B.Y.; Bayari, R. A study on prophylactic doxycycline to reduce the incidence of leptospirosis among paddy field farmers in a coastal district of India. Int. J. Infect. Dis. 2012, 16, E462. [CrossRef]

29. Chusri, S.; McNeil, E.B.; Hortiwakul, T.; Charernmak, B.; Sritrairatchai, S.; Santimaleeworagun, W.; Pattharachayakul, S.; Suksanan, P.; Thaisomboonsuk, B.; Jarman, R.G. Single dosage of doxycycline for prophylaxis against leptospiral infection and leptospirosis during urban flooding in southern Thailand: A non-randomized controlled trial. J. Infect. Chemother. 2014, 20, 709-715. [CrossRef]

30. Alikhani, A.; Salehifar, E.; Zameni, F.; Rafiei, A.; Yazdani-Charati, J.; Delavaryan, L.; Akbari, A.; Babamahmoudi, F. Comparison of azithromycin vs. doxycycline prophylaxis in leptospirosis, a randomized double blind placebo-controlled trial. J. Infect. Dev. Ctries. 2018, 12, 991-995. [CrossRef]

31. Charan, J.; Saxena, D.; Mulla, S.; Yadav, P. Antibiotics for the treatment of leptospirosis: Systematic review and meta-analysis of controlled trials. Int. J. Prev. Med. 2013, 4, 501-510.

32. Brett-Major, D.M.; Coldren, R. Antibiotics for leptospirosis. Cochrane Database Syst. Rev. 2012, CD008264. [CrossRef] [PubMed]

33. Niwattayakul, K.; Kaewtasi, S.; Chueasuwanchai, S.; Hoontrakul, S.; Chareonwat, S.; Suttinont, C.; Phimda, K.; Chierakul, W.; Silpasakorn, S.; Suputtamongkol, Y. An open randomized controlled trial of desmopressin and pulse dexamethasone as adjunct therapy in patients with pulmonary involvement associated with severe leptospirosis. Clin. Microbiol. Infect. 2010, 16, 1207-1212. [CrossRef] [PubMed]

34. Rodrigo, C.; Lakshitha de Silva, N.; Goonaratne, R.; Samarasekara, K.; Wijesinghe, I.; Parththipan, B.; Rajapakse, S. High dose corticosteroids in severe leptospirosis: A systematic review. Trans. R. Soc. Trop. Med. Hyg. 2014, 108, 743-750. [CrossRef] [PubMed]

35. Trivedi, S.V.; Vasava, A.H.; Patel, T.C.; Bhatia, L.C. Cyclophosphamide in pulmonary alveolar hemorrhage due to leptospirosis. Indian J. Crit. Care Med. 2009, 13, 79-84. [CrossRef] [PubMed]

36. Bal, A.M. Use of ciprofloxacin for treating leptospirosis-need for clinical trials. J. Med. Microbiol. 2005, 54, 907. [CrossRef] 